\title{
Congested transport at microscopic and macroscopic scales
}

\author{
B. Maury*
}

\begin{abstract}
This note addresses mathematical issues raised by congestion constraints in transport equations that arise in the modeling of crowd motion or more general active entities. We address in particular the differences between the microscopic and the macroscopic settings.
\end{abstract}

2010 Mathematics Subject Classification. 34G25, 35R70, 70E55.

Keywords. Continuity equation, crowd motion, optimal transportation, Wasserstein space, granular flows, non-smooth analysis.

\section{Introduction}

We are interested here in the role played by congestion constraints in some evolution processes of active entities, and in the way it modifies the mathematical properties of the associated equations. By congestion we mean the following: at the microscopic level, entities are represented by moving geometrical objects (like discs, or spheres), and congestion simply means that two entities may not occupy the same space at the same time; at the macroscopic level, the population is represented by a density that cannot increase above a prescribed maximal value. Most part of the approach presented here has been developped in the context of human crowd motions, but it may as well apply to other entities like swimming cells (we refer to [25] for experiments on bacteria), insects ([21]), or larger animals like sheeps $([11])$. Strategies to account for congestion can be classified according to criteria that are standard in particle physics or granular flow modeling: Soft Sphere / Hard Sphere approaches or, similarly, Molecular Dynamics / Contact Dynamics. In the first (soft) approach, congestion is treated in a smooth way, typically by adding a repulsion force that acts whenever entities become close to each other. If properly tuned, this force prevents a full overlapping of entities (see e.g. [13], or [12] for an application of this strategy in the context of crowd motion modeling). In the macroscopic setting it may take different forms. The one that corresponds to short-range repulsion forces consists in adding some sort of pressure within the population, seen here as a compressible fluid (see [7]). Depending on the context and on the underlying physical reality, other choices are possible and justified. It may for example consist in directly inhibiting the mechanism that is identified as the cause of concentration (see [8], where the chemotactic velocity is

*The author is partially supported by ANR Project Isotace (ANR-12-MONU-0013). 
assumed to decrease where local density increases), reducing the desired velocity in crowded areas, in the context of crowd motion ([14, 15]), adding nonlinear diffusion terms (see e.g. [2]), or incorporating correction terms that nonlocally depend on the crowd distribution, in order to deviate the trajectory of people to avoid overcrowded areas $([22])$.

Let us also mention higher order models that incorporate inertial or delay effects, leading to an evolution equation for velocities, see [5] or [27].

The present text focuses on the alternative approach, that is accounting for congestion constraints in a hard way. At the microscopic level, it consists in strictly forbidding the overlapping of entities (identified as rigid objects), whereas at the macroscopic level, a set a feasible densities will be defined by prescribing a maximal value for the density. We shall investigate here how these constraints are likely to affect the evolution process that is considered, in the framework of very crude modeling assumptions: entity tendencies correspond to a pure transport process (by the velocity that each of them would like to have if it were alone) that is prevented and modified by congestion.

The present paper puts in perspective different works that have been carried out by the author and various collaborators, it aims in particular at identifying the similarities between the microscopic and macroscopic settings, and more importantly to highlight their deep discrepancies. It focuses on the hard congestion problem, but most remarks that we make here to distinguish the two scales of description are applicabled to soft congestion. Sections 2 and 3 present in parallel the models at both scales, and highlight their formal analogies, while Section 4 asserts their differences, and details how those differences at the mathematical level affect the very behavior of the associated evolution models.

\section{Congested transport at both scales}

2.1. Microscopic model. The first problem we shall consider consists in finding a trajectory $t \longmapsto q(t)$ in a euclidean space, starting from a given initial value, verifying

$$
\frac{d q}{d t}=P_{C_{q}} U(q)
$$

where $U(q)$ is a "spontaneous" velocity field, and $C_{q}$ is a set of feasible velocities. The instantaneous projection on $C_{q}$ is intended to force $q$ to remain in a given set $K$ of feasible configurations.

The crowd motion microscopic model (firstly introduced in [17]) fits in this framework: individual are identified with rigid discs of radius $r>0$ evolving in a two-dimensional space, so that the position vector $q=\left(q_{1}, \ldots, q_{N}\right) \in \mathbb{R}^{2 N}$ is subject to remain in

$$
K=\left\{q \in \mathbb{R}^{2 N}, \quad D_{i j}(q)=\left|q_{j}-q_{i}\right|-2 r \geq 0 \quad \forall i<j\right\} .
$$

Feasible velocities are such that, when there is a contact $\left(D_{i j}=0\right)$, the distance may not be further reduced. Thus, the set of feasible velocities is straightforwardly 
defined as

$$
C_{q}=\left\{v \in \mathbb{R}^{2 N}, D_{i j}(q)=0 \Rightarrow G_{i j} \cdot v \geq 0\right\},
$$

where $G_{i j}=\nabla D_{i j}$. Given a vector of desired velocities $U=\left(U_{1}, \ldots, U_{N}\right) \in \mathbb{R}^{2 N}$ (where $U_{i}$ is the velocity that individual $i$ would like to have), the model takes the form (2.1). In the present case, the projection on $C_{q}$ can be put in a saddle-point form

$$
\begin{aligned}
u-\sum_{i \sim j} p_{i j} G_{i j} & =U, \\
-G_{i j} \cdot u & \leq 0 \quad \forall i \sim j, \\
p & \geq 0 \\
\sum p_{i j}\left(G_{i j} \cdot u\right) & =0 .
\end{aligned}
$$

where each $p_{i j}$ accounts for the constraints between $i$ and $j$, and can be seen as an interaction repulsive force that acts against overlapping. Introducing the matrix $B$, each line of which expresses a constraint pertaining to a couple in contact, we obtain

$$
\mid \begin{array}{ll}
u+B^{\star} p & =U \\
B u & \leq 0 \\
p & \geq 0 \\
p \cdot B u & =0
\end{array}
$$

It formally takes the form of a unilateral Darcy problem (see (2.8) below, which corresponds to a standard Darcy problem in a PDE setting). The fact that $B^{\star}$ can be seen as a discrete gradient is clear from the one dimensional case, with $N$ persons in a row (like in Fig. 2a). Applied to a pressure vector, it computes the (opposite of the) resultant force on $i$, that is $p_{i, i+1}-p_{i-1, i}$. We shall see in Section 4.2 that the interpretation of $B^{\star}$ as a gradient is more tedious in the twodimensional setting, and that the richness of the model actually lies in this very discrepancy between $B^{\star}$ and a proper discrete gradient operator, like the ones that are obtained by consistent space discretization.

2.2. Macroscopic model. The very same principles can be applied at the macroscopic level, as initially proposed in [18]. The population is described by a measure density $\rho$ supported in a domain $\Omega$, that aims at being transported according to a given velocity field $U$ (desired velocity), yet subject to remain in a set of feasible densities

$$
\widehat{K}=\left\{\rho \in L^{1}(\Omega), \quad 0 \leq \rho \leq 1 \quad \text { a.e. }, \quad \int_{\Omega} \rho(x) d x=1\right\} .
$$

A velocity is considered feasible if it does not lead to a violation of the constraint above, therefore it consists in velocities that do not overconcentrate the density in zones that are already saturated. Unformally said, it requires $\nabla \cdot u \geq 0$ wherever $\rho=1$. This can be formalized in a dual way :

$$
C_{\rho}=\left\{v \in L_{\rho}^{2}(\Omega), \quad \int_{\Omega} v \cdot \nabla p \leq 0 \quad \forall p \in H_{\rho}^{1}, \quad p \geq 0 \quad \text { a.e. }\right\}
$$


where the set $H_{\rho}^{1}$ of pressure test functions is defined as

$$
H_{\rho}^{1}=\left\{p \in H^{1}(\Omega), p(1-\rho)=0 \text { a.e. }\right\} .
$$

The macroscopic problem (macroscopic counterpart of (2.1)) simply writes

$$
\begin{aligned}
\frac{\partial \rho}{\partial t}+\nabla \cdot(\rho u) & =0 \\
u & =P_{C_{q}} U
\end{aligned}
$$

where the continuity equation is meant in a weak sense (see e.g. [23]), and the projection on $C_{q}$ corresponds to the $L^{2}$ norm. The formal similarity with the microscopic approach is underlined by the saddle-point formulation of the projection problem. Let us define the essential saturated zone as the largest open set $\omega \subset \Omega$ such that $\rho(x)=1$ for a.e. $x \in \omega$. The projection problem takes the form: Find $(u, p) \in L^{2}(\omega) \times H_{0}^{1}(\omega)$ such that

$$
\begin{aligned}
u+\nabla p & =U \text { in } \omega \\
-\nabla \cdot u & \leq 0 \quad \text { in } \omega \\
p & \geq 0 \quad \text { in } \omega \\
\int_{\omega} u \cdot \nabla p & =0
\end{aligned}
$$

that is the macroscopic counterpart of system (2.5). It takes the form of a Darcy problem, that is commonly used to describe the flow of an incompressible fluid in a porous medium (see e.g. [4]). The latter problem provides the effective velocity in $\omega$ only. The overall velocity $u$ is obtained by extending $u_{\mid \omega}$ with $U$ (the actual velocity is obviously the desired one outside the saturated zone).

Proposition 2.1. Problem (2.8) has a unique solution $(u, p) \in L^{2}(\omega)^{2} \times H_{0}^{1}(\omega)$.

Proof. This is a straightforward consequence on the fact that the operator

$$
\widehat{B}: v \in L^{2}(\omega)^{2} \longmapsto-\nabla \cdot u \in H^{-1}(\omega),\langle\widehat{B} v, p\rangle=\int v \cdot \nabla p
$$

is surjective, thanks to Poincaré inequality in $H_{0}^{1}(\Omega)$, so that $\widehat{B}^{\star}$ is one-to-one and has closed range. As a consequence, the polar cone to the set of feasible velocities $C_{q}$ can be written

$$
C_{q}^{\circ}=\left\{w \in L^{2}(\omega)^{2}, \int_{\Omega} w \cdot v \leq 0 \quad \forall v \in C_{q}\right\}=\left\{\nabla p, \quad p \in H_{0}^{1}(\omega), \quad p \geq 0\right\},
$$

hence the existence and uniqueness of a saddle-point $(u, p)$.

Whether this macroscopic model could be properly obtained through a micromacro limit from the first will be addressed, and in some way answered, in a negative way in Section 4. 


\section{Well posedness of the time evolution problems}

3.1. Microscopic model. The evolution problem (2.1) consists in finding a continuous path $t \mapsto q(t) \in \mathbb{R}^{2 N}$

$$
\frac{d q}{d t}+\partial I_{K}(q) \ni U(q) \quad \text { a.e. in }[0, T], q(0)=q_{0},
$$

where $I_{K}(q)=0$ as soon as $q \in K$ (equal to $+\infty$ otherwise), and $\partial I_{K}$ is the Fréchet subdifferential, defined for $q \in K$ by

$$
\begin{array}{r}
\partial I_{K}(q)=\left\{w, I_{K}(q)+h \cdot w \leq I_{K}(q+h)+o(h) \quad \forall h\right\} \\
=\{w, h \cdot w \leq o(h) \quad \forall h \text { s.t. } q+h \in K\} .
\end{array}
$$

Proposition 3.1. Let the set of feasible configuration $K$ be defined by (2.2), and let the desired velocity $q \mapsto U(q)$ be defined as a Lipschtitz function of $q$. Equation (3.1) admits a unique continuous solution over $[0, T]$.

Proof. The proof mainly relies on a time-discretization scheme, inspired by the socalled catchin-up algorithm initially introduced in [20] to build discrete solutions of sweeping processes. Let $\tau>0$ be a time step, the sequence $\left(q_{\tau}^{n}\right)$ is built according to

$$
\mid \begin{aligned}
& \tilde{q}^{n+1}=q^{n}+\tau U\left(q^{n}\right), \\
& q^{n+1}=P_{K} \tilde{q}^{n+1},
\end{aligned}
$$

Although the set $K$ of feasible configurations is not convex, it can be shown to be prox-regular, which essentially means that projection on it is well-defined in a neighborhood of its boundary (see $[17,19]$ and the next section). Thus, for $\tau$ sufficiently small, the projection of $\tilde{q}^{n+1}$ on $K$ is well defined. The collection of discrete solutions can be interpolated, which forms a piecewise affine path $t \longmapsto q_{\tau}$. This approximate solution uniformly converges to a solution of (3.1) (see [17] for details).

3.2. Macroscopic model. The macroscopic problem (2.7) has the general form

$$
\frac{\partial \rho}{\partial t}+\nabla \cdot(\rho u(\rho))=0
$$

where $u(\rho)$ is a velocity field, the values of which depend on the density $\rho$ overall the domain, and not only at the considered point. The mapping $\rho \mapsto u(\rho)$ is far from being smooth, and the native regularity of the velocity itself is simply $L^{2}$, so that standard theory is not applicable. An alternative approach was proposed in $[18,19]$, based on optimal transportation. It consists in extending Moreau's approach, that was initially dedicated to Hilbert spaces, to measures. The scheme reads as follows:

$$
\mid \begin{aligned}
\tilde{\rho}^{n+1} & =(\mathrm{Id}+\tau U) \rho^{n}, \\
\rho^{n+1} & =P_{\widehat{K}} \tilde{\rho}^{n+1},
\end{aligned}
$$


where the projection is meant in the square Wasserstein sense, i.e. according to the distance defined by

$$
W_{2}(\mu, \nu)^{2}=\arg \min _{\Lambda_{\mu, \nu}} \int_{\Omega}|T(x)-y|^{2} d \mu(x),
$$

where $\Lambda_{\mu, \nu}$ is the set of all those measurable maps that push $\mu$ forward to $\nu$. The minimum above is attained whenever $\mu$ does not charge zero-measure sets (which holds in the situation we consider here), see [28] or [24].

Proposition 3.2. Let $\Omega$ be a bounded convex domain, $\rho_{0} \in \widehat{K}$ an initial density, and let $U$ be a Lipschitz velocity field, such that $U$ points inward the domain on $\partial \Omega$. We consider $t \in[0, T] \longmapsto \rho_{\tau}(t) \in \widehat{K}$ (defined by (2.6)) the path obtained by geodesic interpolation between the discrete densities obtained by application of the scheme $(3.4)$, and by $u_{\tau}$ the associated velocity. Then the couple $\left(\rho_{\tau}, u_{\tau}\right)$ converges to a solution $(\rho, u)$ of Problem (2.7).

Proof. We refer to [19] for a detailed proof of this convergence. Let us simply address here a particular issue raised by the projection step (second line of (3.4)), that enlight the fact that this macroscopic model is not a straight extension of the microscopic model. Existence of a minimizer comes from standard compactness arguments. As for uniqueness, $\widehat{K}$ is convex in the geodesical (or Mc Cann) sense: for any two measures $\rho_{0}$ and $\rho_{1}$ in $\widehat{K}$, and $T$ an optimal map from $\rho_{0}$ to $\rho_{1}$, then

$$
\rho_{t}=((1-t) \operatorname{Id}+t T)_{\sharp} \rho_{0} \in \widehat{K} \quad \forall t \in[0,1] .
$$

Yet, as pointed out in [3], the functional $\rho \mapsto W_{2}(\tilde{\rho}, \rho)^{2}$ is not geodesically convex. Uniqueness can nevertheless be obtained by using the fact that the latter functional is convex along generalized geodesics. In the present situation, such geodesics are defined as follows: consider $\rho_{0}$ and $\rho_{1}$ in $\widehat{K}, \tilde{\rho}$ the measure that is to be projected, and $T_{0}$ and $T_{1}$ optimal transport maps from $\tilde{\rho}$ to $\rho_{0}$ and $\rho_{1}$, respectively. The generalized geodesic is obtained by interpolating the transport maps, i.e.

$$
\rho_{s}=\left((1-s) T_{0}+s T_{1}\right)_{\sharp} \tilde{\rho} .
$$

It can be checked that $\widehat{K}$ is still convex with respect to this new definition of geodesics (see [18]), and uniqueness follows.

\section{Micro-macro discrepancies}

The microscopic and the macroscopic models express the very same principle at different levels of description: the actual velocity is the closest (in a least square sense) to the desired velocity, among all feasible velocities. They futhermore present formal analogies, up to the saddle-point formulations of the minimization problem that defines the instantaneous velocities (Systems (2.5) and (2.8)). Yet, those analogies covers deep discrepancies that are described in this section. 
4.1. Geometrical properties of the Wasserstein space. This section addresses some micro-macro issues linked with the geometrical properties of the sets of feasible configurations: $K$ defined by (2.2) for the microscopic situation, and its macroscopic counterpart $\widehat{K}$ defined by (2.6). Although they obviously play and equivalent role, they have very distinct properties from a geometrical standpoint.

At the microscopic level, $K$ is the set of hard discs configurations without overlapping. It is obviously non convex, and the euclidean projection on this closed set is not uniquely defined outside a thin neighborhood. The notion of $\eta$ prox regularity quantifies this property (see e.g. [26]): $K$ is said to be uniformly $\eta$-prox regular whenever

$$
\forall q \in \partial K, \quad \forall v \in \partial I_{k}(q) \text { with }|v|=1, q=P_{K}(q+\eta v),
$$

where $\partial I_{K}$ is defined by (3.2). It can be proven (see [17]) that $K$ (defined by (2.2)) is indeed $\eta$-prox regular, with a constant $\eta$ that goes to 0 when $N \rightarrow+\infty$ together with $r \rightarrow 0$.

This degeneracy suggests a singular behavior in the micro-macro limit. Indeed, the macroscopic set of feasible density $\widehat{K}$ does not present the characteristic of a prox-regular set. Let us first remark that it has empty interior (for the Wasserstein metric), so that any density $\rho$ that never saturates the constraints belongs to $\partial \widehat{K}$, while it is obviously the projection on $\widehat{K}$ of itself only. Even when one considers densities that saturate the constraint on their support, uniform $\eta$-prox regularity is immediately ruled out. Consider for example a density that is the indicator function of a collection of non-overlapping discs of common radius $1 / \sqrt{N}$ :

$$
\rho_{N}=\frac{1}{\pi} \sum_{i=1}^{N} \mathbb{1}_{B\left(x_{i}, 1 / \sqrt{N}\right)} .
$$

Among all those measures that projects on $\widehat{K}$ at $\rho_{N}$, the most remote is a sum of Dirac masses supported by the collection of disc centers, and the corresponding distance scales like $1 / \sqrt{N}$, which goes to 0 as $N$ goes to $+\infty$.

In the Hilbertian setting, the projection on such a set (that rules out any uniform prox-regularity) would not be properly defined, even in a close neighborhood of the set. Yet, as mentioned in the proof of Proposition 3.2, the distance from a measure $\rho$ to $\widehat{K}$ is always attained at a unique point in $\widehat{K}$, no matter how far $\rho$ is from $\widehat{K}$. The apparent contradiction between the properties of $P_{K}$ and $P_{\widehat{K}}$ are actually due to the fact that the Wasserstein setting corresponds to a Lagrangian description of particles up to permutations. It leads to a puzzling property, that we will illustrate by a simple example: in some cases, considering a density $\rho \in \widehat{K}$, and a vector field that belong to the subdifferential (in the wasserstein sense) of $I_{\widehat{K}}$ (such a velocity field can be seen as directed along the "outward normal direction" of $\widehat{K})$, the curve $\rho_{t}=(\mathrm{Id}+t v)_{\sharp} \rho$ starts indeed moving straight away from $\widehat{K}$, then turns back toward $\rho$, and re-enters $\widehat{K}$ at $\rho$. Let us make it straight that $(\operatorname{Id}+t v)_{\sharp} \rho$ does not represent a solution to the transport equation with the Eulerian velocity field $v$ and initial density $\rho$, but rather the one-shot push-forward (image measure) 

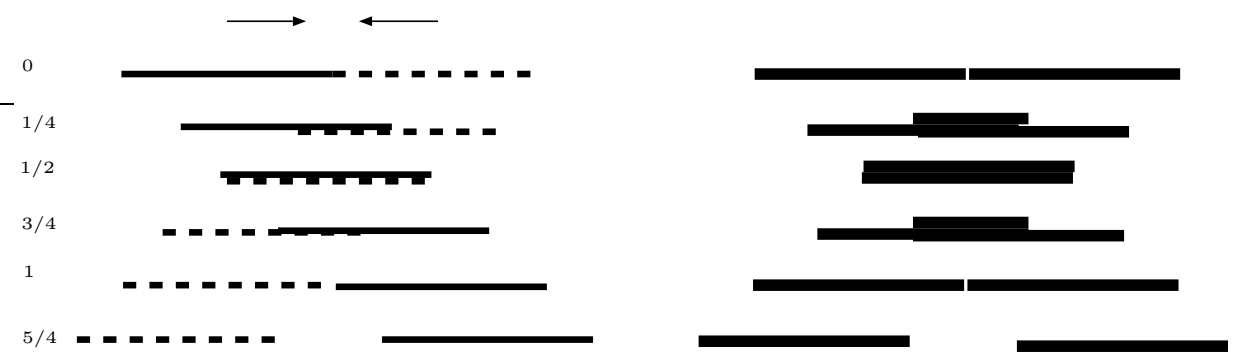

Figure 1. Crossing of entities, Lagrangian and Wasserstein viewpoints

of $\rho$ by the map

$$
x \longmapsto(\operatorname{Id}+t v)(x)=x+t v(x) .
$$

The example is the following: in the one-dimensional setting, one considers two individuals identified to rigid segments of length one, initially in contact, we consider a velocity field $(1,-1)$ that belongs to $\partial K$ (see $(5.1)$ below), and we consider the microscopic path $q_{t}=q+t v$. For $t \in\left[0,1 / 2\left[\right.\right.$, the projection of $q_{t}$ on $K$ is $q_{0}=q$. For $t=1 / 2$ the two segments coincide, and the projection is not unique (the distance is attained for $q$ and for the configuration obtained by interchanging 1 and 2, that are distinct since the description is fully Lagrangian). For larger $t$, the projection is well defined, but it is not the starting point $q$. The right-hand side of the figure represents the Wasserstein version of this situation, with the very same velocity field. Segments are represented in the same way, since "particles" are indistinguishable. For $t \in[0,1]$, the projection of $\rho_{t}=(\mathrm{Id}+t v)_{\sharp} \rho$ is well-defined, with a unique projection that is the initial configuration. The path $\rho_{t}$, that follows a velocity field that point outward of $K$, actually moves away from $\rho$ in a first phase, but then returns back to its very initial position, unlike in the microscopic situation.

4.2. Discrete counterparts of the differential operators. We describe here the discrepancies between $B, B^{\star}$, and $B B^{\star}$, discrete analogs of gradient, divergence, and Laplacian operators, respectively, and their macroscopic counterparts.

Discrete divergence. As already mentioned, the matrix $B$ that expresses the non overlapping constraints (see Equation (2.5)) is the discrete counterpart of the opposite of the divergence operator in the macroscopic Darcy equation (2.8). Let us denote by $N_{c}$ the number of contacts. Each row of $B \in \mathcal{M}_{N_{c}, 2 N}(\mathbb{R})$ corresponds to a contact between two discs $i$ and $j$ :

$$
\left(0, \ldots, 0, e_{i j}, 0, \ldots, 0,-e_{i j}, 0, \ldots, 0\right) \in \mathbb{R}^{2 N},
$$

where $e_{i j}=\left(q_{j}-q_{i}\right) /\left|q_{j}-q_{i}\right|$ is the unit vector between centers. In the onedimensional setting, $B u \leq 0$ prevents inter-center distances to decrease, thus it is a straight counterpart of divergence non-negativity. In the two-dimensional setting, the constraint highly depends on the disc arrangement. In the cartesian case 


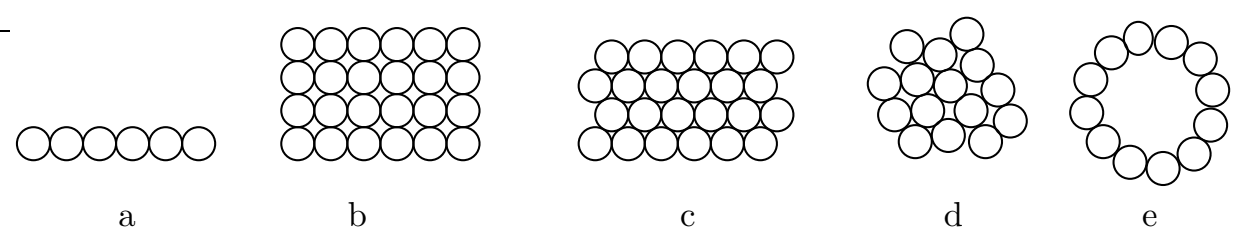

Figure 2. Typical disc arrangments

(Fig. 2b), $B u \leq 0$ imposes the same kind of constraint along $x$ and $y$ directions, independently, which is much stronger that imposing a local non-negative divergence like in the macroscopic situation. The constraints are even stronger for the triangular lattice (Fig. 2c): the monotone character of the velocity is imposed in three different directions. In the general case (unstructured cluster like in (Fig. 2d)), the contraints are imposed along directions that depend on the local arrangement on discs. Those examples show that the discrete constraint $B u \leq 0$ is much stronger than its macroscopic counterpart $-\nabla \cdot u \leq 0$.

Discrete gradient. Such a discrepancy can be formulated in a dual way by considering the matrix $B^{\star}$ defined in Section 2 , whose role is to transfer Lagrange multipliers (interaction repulsive forces) onto velocity corrections (see Equation (2.5)). This matrix plays the role of the gradient in the macroscopic Darcy equation (2.8). This analogy is fully relevant in the one-dimensional setting. In particular, if one considers a cluster of aligned discs (see Fig. 2a), a uniform pressure fields induces no effect on the discs (except at both ends of the row). This remains true in the two-dimensional setting in very particular, structured situations, like the ones represented in Fig. 2b and Fig. 2c (cartesian and triangular lattice), but this property is lost for clusters with no symmetries (like in Fig. 2d). In the particular configuration represented in Fig. 2e, a uniform pressure field induces a centrifugal force field. In case all discs tend to reach the center of the circle, this allows for a static state to exist, with a exact balance between the desired velocity field and $-B^{\star} p$. This example can be interpreted as a static jam, and we shall see than such jams have no equivalent in the macroscopic setting.

The fact that discs generically have more than 4 neighbors in congested situations induces a mathematical degeneracy that has deep consequences from the modeling standpoint. Consider a large population of $N$ discs arranged according to the triangular lattice (Fig. 2c). The number of primal degrees of freedom (centers of discs) is $2 N$, whereas the number $N_{c}$ of contacts is of the order $3 N$, so that $B^{\star}=\mathcal{M}_{2 N, N_{c}}(\mathbb{R})$ is highly singular: the pressure is not defined in a unique way. Let us note though that, in spite of this degeneracy in terms of pressure uniqueness, the solution set is bounded, as expressed by Proposition 4.2. The core of this property relies on the following lemma, which asserts that non trivial degenerate pressure fields (i.e. such that $B^{\star} p=0$ ) always contain pressures with opposite signs.

Lemma 4.1. Let $q \in K$ be given, $B \in \mathcal{M}_{N_{c}, 2 N}(\mathbb{R})$ the associated constraint 

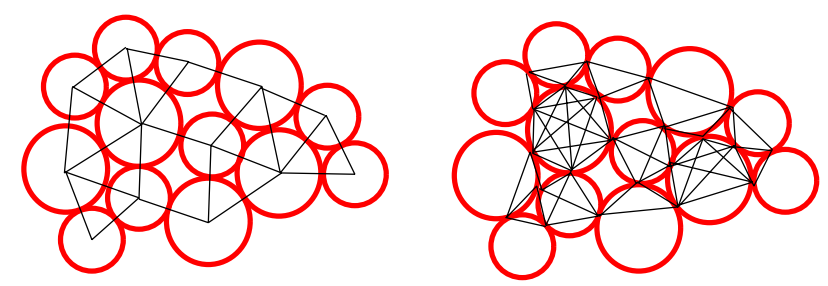

Figure 3. Primal and dual networks

matrix, then $\operatorname{ker} B^{\star} \cap \mathbb{R}_{+}^{N_{c}}=\{0\}$.

Proof. Let $p$ be such that $B^{\star} p=0$, with $p \geq 0$. Consider a connected sub-cluster of $q$ (a connected maximal subset of discs in contact), like the one represented in Fig. 2d. We denote by $J$ the corresponding set of indices. Consider now the convex-hull of centers $\left(q_{i}\right)_{i \in J}$, and pick one $q_{i}$ that is an extremal point of the convex hull. By Hahn Banach's Theorem, $q_{i}$ can be separated from the convex hull of the remaing centers, by an hyperplane normal to some vector $w$. Now write the force balance for $q_{i}$ along direction $w$ :

$$
\sum_{j \sim i} p_{i j} e_{j i} \cdot w=0
$$

By construction $e_{j i} \cdot w>0$ for all $j$ in contact with $i$, so that all pressures involving $i$ are equal to zero. Applying the same approach recursively makes it possible to eliminate extremal points one after the other, thus the pressure is identically 0.

Proposition 4.2. Let $q \in K$ and $U \in \mathbb{R}^{2 N}$ be given, $u=P_{C_{q}} U$. Then the solution set for pressure, i.e. the set $\Lambda$ of all those pressure fields $p \in \mathbb{R}_{+}^{N_{c}}$ such that $(u, p)$ is a solution to (2.5), is bounded.

Proof. The solution set writes

$$
\Lambda=\left\{p \in \mathbb{R}^{N_{c}}, \quad B^{\star} p=U-u, p \geq 0\right\} .
$$

If a sequence $\left(p_{n}\right)$ of pressures in $\Lambda$ goes to infinity, then, up to a subsequence, $p_{n} /\left|p_{n}\right|$ converges to $p \in \operatorname{ker} B^{\star} \cap \mathbb{R}_{+}^{N_{c}}$, that is necessarily 0 .

Discrete Laplacian. The previous remarks explain a difference in the behaviour of the two models, that is highly significant if one considers evacuation processes. In the macroscopic setting, the congested zone upstream the exit has the typical shape represented in Fig. 4 (left). The congested zone is denoted by $\omega$, and the desired velocity field points toward the exit, and verifies $\nabla \cdot U<0$. The pressure field is then solution of a Poisson problem

$$
-\Delta p=-\nabla \cdot U>0
$$


with homogeneous Dirichlet boundary conditions on the upper free boundary, and on the exit, and homogeneous neuman condition on the wall (no normal velocity). By the maximum principle, the pressure is nonnegative in $\omega$, so that

$$
u \cdot n=U \cdot n-\partial p / \partial n \geq U \cdot n,
$$

where $n$ is the outward normal on the exit boundary. As a consequence, the crowd exits the room faster than it would if there were non congestion. This feature contradicts experimental evidence, in particular the so-called capacity drop phenomenon: beyond a certain threshold, congestion tends to decrease the evacuation speed (see e.g. [6]). This model could be corrected by imposing a flux reduction in case of upstream congestion, in the spirit of what is proposed in [1], to force the capacity drop phenomenon, but such a ingredient does not really model, neither explains, the considered phenomenon.

On the contrary, the microscopic model natively reproduces this behavior, because of the aforementioned nonstandard character of the underlying discrete operators. Indeed, there exist static configurations with all individuals pointing toward a door that is significantly wider than the widest individual, where the desired velocity is exactly balanced by the action of a positive pressure field. Such a static jam is represented in Fig. 4 (right). In such a situation, the pressure field $p$ solves (2.5) with $u=0$. The desired velocity field is concentrating, i.e. it tends to reduce the distances, thus by definition $B U>0$. The pressure is then solution to a discrete Poisson-like problem, discrete counterpart of (4.2):

$$
B B^{\star} p=B U
$$

with a positive right hand side. In some structured situations, $B B^{\star}$ is a standard discrete Laplacian. For a 1d row of individuals for example (Fig. 2a), it is the one dimensional discrete Laplace operator with Dirichlet boundary conditions at the ends. The Dirichlet character of boundary conditions expresses the fact that individuals at the end of the row are free to move away from the cluster (i.e. the outside pressure is zero). In the cartesian case (Fig. 2b), this matrix encodes two 1d Laplace operator (along $x$ and $y$ axes). Note the difference with the macroscopic situation: the discrete pressure field does not correspond to a scalar pressure fields, but to a collection of two scalar pressure fields, one for the horizontal direction, one for the vertical one. When the configuration is unstructured, like in (Fig. 2d), the situation is more complex. The operator $B B^{\star}$ is defined on the networks of contact points, that is dual to the network of disc centers (see Fig. 3 (right)).

Considering a pressure field $p=\left(p_{k \ell}\right)$, where $(k, \ell)$ runs over active contacts, the vector $B B^{\star} p$ is defined on the dual network, and the value that corresponds to the contact between $i$ and $j$ is

$$
\sum_{(k, \ell) \sim(i, j)} p_{k \ell} G_{i j} \cdot G_{k \ell},
$$

where $(k, \ell) \sim(i, j)$ means that the two contacts share a common disc. This matrix shares some properties with matrices associated with a resistive network, or 


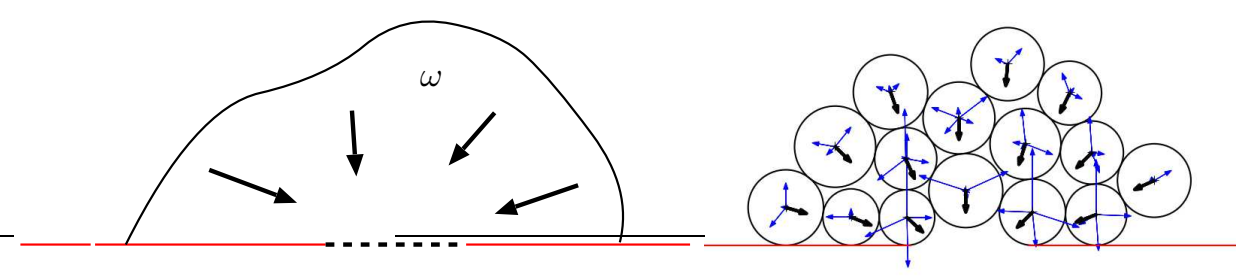

Figure 4. Macroscopic and microscopic jams

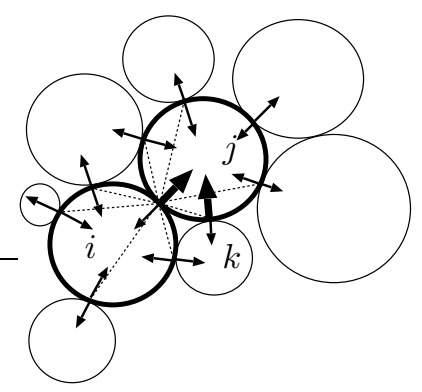

Figure 5. Unstructured stencil

matrices resulting from the space discretization of the Laplace operator by a Finite Difference / Finite Element method, in particular it is symmetric and its diagonal elements are positive. Yet, some extradiagonal coefficients are positive, like in the situation represented in Fig. 5. The element of $B B^{\star}$ that encodes the interaction between $(i, j)$ and $(i, k)$ is $e_{i j} \cdot e_{i k}>0$. The resistive networks associated with this matrix therefore admits negative resistances, so that the maximum principle does no longer hold, ruling out the arguments that we used in the macroscopic situation, to assert that individuals escape faster than they would if they were alone. Indeed, the microscopic model reproduces static jams, like the one represented in Fig. 4. In this situation, all individuals tend to reach the exit, but positive pressures create upstream correction terms that exactly balance the desired velocities. One may qualify this phenomenon as some sort of inverse surface tension: when the border of the cluster presents a locally concave shape, a positive pressure field between discs induces a force that points inward the cluster.

\section{Gradient flow structure of the evolution process}

Both models may be formulated in a gradient flow framework, in case of evacuation processes. As for the microscopic one, it is natural to define the desired velocity of an individual centered at $q_{i}$ as $-\nabla D\left(q_{i}\right)$, where $D(\cdot)$ is the geodesic distance to the exit. The latter quantity can be considered as an individual dissatisfaction. 
The global dissatisfaction can then be defined as the sum of individual ones, e.g.

$$
\Phi(q)=\sum_{i=1}^{N} D\left(q_{i}\right)
$$

Since the set $K$ is pro-regular (i.e. it verifies (4.1) for some $\eta>0$, and the subdifferential of $I_{K}$ is well defined by $(3.2)$ ), it can be checked that the evolution equation (2.1) can be written (see [17])

$$
\frac{d q}{d t}=-\nabla \Phi(q)-\partial I_{K}(q)=-\partial\left(\Phi+I_{K}\right)(q)
$$

that is a gradient flow associated to the dissatisfaction functional $\Phi+I_{K}$.

In the macroscopic setting, the Wasserstein framework makes it possible to exhibit a similar structure based on a macroscopic dissatisfaction function canonically defined as

$$
\widehat{\Phi}(\rho)=\int_{\Omega} D(x) \rho(x) d x .
$$

Its subdifferential is defined as the set of all those fields $w \in L_{\rho}^{2}(\Omega)$ such that

$$
\widehat{\Phi}(\rho)+\int_{\Omega} w \cdot v \rho(x) d x \leq \widehat{\Phi}\left(T_{\sharp} \rho\right)+o(\|T-\operatorname{Id}\|),
$$

for all transport maps $T$. As detailed in [18], the evolution takes the expected form of a gradient flow in the Wasserstein space:

$$
\begin{array}{r}
\frac{\partial \rho}{\partial t}+\nabla \cdot(\rho u)=0 \\
u \in-\partial\left(\widehat{\Phi}+I_{\widehat{K}}\right)(\rho) .
\end{array}
$$

Again, the formal analogy hides deep differences in terms of behavior. In case of an evacuation like in Fig. 4 (left), the functional $\widehat{\Phi}+I_{\widehat{K}}$ is geodesically convex, and there is no density distribution such that $0 \in-\partial\left(\widehat{\Phi}+I_{\widehat{K}}\right)$, i.e. no equilibrium point that would correspond to a static jam. On the contrary, at the microscopic level, a situation like in Fig. 4 (right) correspond to a critical point of the nonsmooth functional $\Phi+I_{K}$. The latter dissatisfaction functional is not convex, and it admits local minima that correspond to static jams. Because of the non smooth character of the functional, stability of such jams cannot be asserted by standard tools, but the generalized Hessian that is introduced in [10] makes it possible to verify that some of those jams that spontaneously appear actually correspond to local minima, and are therefore stable.

\section{Conclusion}

The previous considerations show that some features of the microscopic model are not captured by the macroscopic one. This discrepancy between the two models 
is not a contradiction: the macroscopic model has not been obtained as a rigorous limit of the microscopic one, but rather by simply expressing the same principles at the macroscopic level : the actual velocity field is the closest to the desired velocity field in the set of feasible fields. At the microscopic level, feasibility calls for monotonicity constraints in various directions (e.g. 3 directions for the triangular lattice, as detailed in Section 4.2), whereas, at the microscopic level, the divergence free constraint is scalar. The constraints are much stronger for a hard-sphere collection than for a continuous, deformable medium, solely based on local density. A continuous model fully respectful of microscopic features would necessarily rely on extra quantities reflecting the local arrangement of grains, as illustrated by the several discrepancies listed in the previous section (we also refer to [19], Section 5, for a further description of the obstacles that prevent from elaborating a rigorous macroscopic limit of a hard sphere system in highly packed situations). Up to our knowledge, there does not exist any micro-macro framework that allows to recover at the macroscopic scale the features of the microscopic hard-sphere model, in highly congested situations.

\section{References}

[1] B. Andreianov, C. Donadello, U. Razafison, M. D. Rosini, Riemann problems with non-local point constraints and capacity drop, Mathematical Biosciences and Engineering, Volume 12, Issue 2, pp. 259 - 278, April 2015.

[2] D. Alexander, I. Kim, Y. Yao, Quasi-static evolution and congested crowd transport. Nonlinearity, 27, 1-36, 2014.

[3] L. Ambrosio, N. Gigli and G. Savaré, Gradient Flows in Metric Spaces and in the Space of Probability Measures, Lectures in Mathematics, ETH Zürich, Birkhäuser Verlag, Basel, 2005.

[4] J. Bear, Dynamics of Fluids in Porous Media, Dover Publications, New York, 1988.

[5] N. Bellomo and C. Dogbé, On the modelling crowd dynamics from scaling to hyperbolic macroscopic models, Math. Models Methods Appl. Sci. 18 (2008) 1317-1345.

[6] E. M. Cepolina, A. Farina, A Pedestrian Movement Model That Takes into Account the Capacity Drop Phenomenon in the Motion of Crowd, Cellular Automata, Volume 6350 of the series Lecture Notes in Computer Science pp 446-454.

[7] P. Degond, L. Navoret, R. Bon and D. Sanchez, Congestion in a macroscopic model of self-driven particles modeling gregariousness, J. Stat. Phys., 138 (2010), 85-125.

[8] Y. Dolak, C. Schmeiser, The Keller-Segel model with logistic sensitivity function and small diffusivity, SIAM J. Appl. Math., 66(1) (2005), 286-308.

[9] J. F. Edmond and L. Thibault, BV solutions of nonconvex sweeping process differential inclusion with perturbation, J. Differential Equations, 226 (2006), 135-179.

[10] S. Faure, B. Maury, Crowd motion from the granular standpoint, Mathematical Models and Methods in Applied Sciences, Vol. 25, No. 3 (2015) 463-493.

[11] A. Garcimartín, J. M. Pastor, L. M. Ferrer, J. J. Ramos, C. Martín-Gómez, I. Zuriguel, Flow and clogging of a sheep herd passing through a bottleneck, Physical Review E 91, 022808 (2015). 
[12] D. Helbing and P. Molnár, Social force model for pedestrian dynamics, Phys. Rev E, 51 (1995), 4282-4286.

[13] D.C. Hong, J.A. McLennan, Molecular dynamics simulations of hard sphere granular particles, Physica A: Statistical Mechanics and its Applications, Volume 187, Issues 1?2, 15 August 1992, Pages 159-171.

[14] R. L. Hughes, A continuum theory for the flow of pedestrian, Transport. Res. Part $B, 36$ (2002), 507-535.

[15] R. L. Hughes, The Flow of Human Crowds, Ann. Rev. Fluid Mech., 35 Annual Reviews, Palo Alto, CA, (2003), 169-183.

[16] R. Jordan, D. Kinderlehrer and F. Otto, The variational formulation of the FokkerPlanck equation, SIAM J. Math. Anal., 29 (1998), 1-17.

[17] B. Maury and J. Venel, A discrete contact model for crowd motion, ESAIM Mathematical Modelling and Numerical Analysis, 45 (2011), 145-168.

[18] B. Maury, A. Roudneff-Chupin and F. Santambrogio, A macroscopic crowd motion model of gradient flow type, Mathematical Models and Methods in Applied Sciences, 20 (2010), 1787-1821.

[19] B. Maury, A. Roudneff-Chupin, F. Santambrogio, J. Venel, Handling Congestion in Crowd Motion Modeling, Networks and Heterogeneous Media, Volume 6, Number 3, September 2011, pp. 485-519.

[20] J.J. Moreau, Evolution problem associated with a moving convex set in a Hilbert space. J. Differential Equations, 26(3):347-374, 1977.

[21] D.R. Parisi, S.A. Soria, R. Josens, Faster-is-slower effect in escaping ants revisited: Ants do not behave like humans, Safety Science 72 (2015) 274-282.

[22] B. Piccoli, F. Rossi, Transport equation with nonlocal velocity in Wasserstein spaces: convergence of numerical schemes, Acta Applicandae Mathematicae, 124, pp. 73-105, 2013.

[23] B. Piccoli and A. Tosin, Time-evolving measures and macroscopic modeling of pedestrian flow, Arch. Rational Mech. Anal. 199 (2011) 707-738.

[24] F. Santambrogio, Optimal Transport for Applied Mathematicians. Birkäuser, NY, 2015.

[25] A. Sokolov, I. S. Aranson, J.O. Kessler, R. E. Goldstein, Concentration dependence of the collective dynamics of swimming bacteria, Phys. Rev. Lett. 15, Vol. 98, 2007.

[26] L. Thibault, Sweeping process with regular and nonregular sets, J. Differential Equations, 193 (2003), 1-26.

[27] M. Twarogowska, P. Goatin, R. Duvigneau, Macroscopic modeling and simulations of room evacuation, Applied Mathematical Modelling, Volume 38, Issue 24, 15 December 2014, Pages 5781?5795.

[28] C. VIllani, Topics in Optimal Transportation, Grad. Stud. Math., 58, AMS, Providence, RI, 2003.

B. Maury, Laboratoire de Mathématiques d'Orsay, Univ. Paris-Sud, CNRS, Université Paris-Saclay, 91405 Orsay cedex, France

\& DMA, École Normale Supérieure, 45 rue d'Ulm, Paris

E-mail: Bertrand.Maury@math.u-psud.fr 